\title{
ASOCIACIONISMO, REDES Y MARKETING EN LA TRANSFORMACIÓN HACIA EL TURISMO EXPERIENCIAL. EL CASO DEL BARRIO DE LAS LETRAS. MADRID
}

\author{
Blanca García Henche* \\ Universidad de Alcalá \\ Erica Salvaj Carrera** \\ Universidad del Desarrollo y Universidad Torcuato Di Tella
}

\section{RESUMEN}

Sobre la base de las tradiciones y el patrimonio cultural, las pequeñas empresas pueden, a través de la organización, la colaboración social y el emprendimiento, aprovechar estos activos y transformar los espacios urbanos. La Asociación de Comerciantes Barrio de las Letras está transformando dicho barrio madrileño en un destino turístico de experiencias. El objetivo de este trabajo es explorar cómo las asociaciones comerciales, las redes de negocios entre pequeñas empresas y el marketing pueden transformar barrios social y económicamente alicaídos en zonas florecientes para el turismo y el comercio.

Palabras clave: Asociacionismo, marketing de turismo de experiencias, eventos comerciales, redes sociales, pequeños comercios, marcas de garantía de calidad.

\section{Associations, networks and the transformation toward experiential tourism marketing. The case of the district Barrio de las Letras. Madrid}

\section{ABSTRACT}

Based on traditions and cultural heritage, small businesses can, through organization, social collaboration and marketing leverage these assets and transform urban spaces into

\section{Recibido: 27 de julio de 2016}

Devuelto para su revisión: 7 de noviembre de 2016

Aceptado: 20 de diciembre de 2016

* Facultad de Ciencias Económicas, Empresariales y Turismo. Universidad de Alcalá. Plaza de la Victoria, 2 , 28802, Alcalá de Henares. MADRID (España).E-mail: Blanca.garcia@uah.es

** Universidad del Desarrollo. Avenida Plaza 680, SANTIAGO (Chile); y Universidad Torcuato Di Tella. Av. Pres. Figueroa Alcorta 7350. BUENOS AIRES (Argentina).E-mail: esalvaj@udd.cl 
attractive tourist destinations. The Small Businesses Association of Barrio de las Letras is an example of how transformation of a decaying neighborhood in Madrid into an experiential tourist destination is possible. The main goal of this paper is to explore how small business associations and networks among entrepreneurs can transform neighborhoods with cultural heritage into thriving and economically attractive touristic and trade areas.

Keywords: Business associations, experiential marketing tourism, social networking, small businesses, brands quality.

\section{INTRODUCCIÓN}

Los cascos históricos de las ciudades, debido a su concentración de pequeños comercios y su patrimonio cultural, no son ajenos a la importancia del asociacionismo y las relaciones sociales, en lo referido a su desempeño y adaptación.

A medida que el modelo tradicional de desarrollo turístico comienza a saturarse, las Administraciones Públicas y la propia industria del turismo deben buscar alternativas que proporcionen una respuesta a una demanda cada vez más exigente y segmentada, que busca autenticidad y experiencias únicas. En la actualidad, adquiere cada vez más importancia el turismo experiencial o emocional. Richards (2004), afirma que el turismo cultural está convirtiéndose en un producto de vivencia en el que la visita se juzga en función de todas las características del lugar y no solo por su valor cultural.

En este contexto, se ha considerado conveniente analizar como el asociacionismo y las redes sociales de colaboración entre pequeños negocios permiten la creación de un sello de calidad e identificativo de determinados destinos turísticos, que sirva para calificar y distinguir a diferentes zonas de las cascos históricos de las ciudades, además de poner en valor el patrimonio histórico (material e inmaterial), cultural y social de dichos barrios.

El presente artículo recoge los resultados de un proyecto que analiza los principales cambios que se han producido en el sector comercial y turístico en el Barrio de las Letras de Madrid, desde el surgimiento de la estructura de la red de emprendedores del barrio, la creación de la asociación y marca "Barrio de las Letras" y el desarrollo de las estrategias de comercialización de dicho destino turístico como destino de experiencias.

El artículo se organiza de la siguiente manera: en primer lugar se revisa la literatura sobre las relaciones sociales y su rol en la transformación económica de los espacios geográficos. En segundo lugar se desarrollan los conceptos de marketing y turismo experiencial. Seguidamente, se describe la metodología y los datos. En cuarto lugar, se presenta el caso del Barrio de las Letras. A continuación, se presentan los resultados, que incluyen la descripción de la Asociación de comerciantes del Barrio de las Letras, las estrategias de marketing experiencial llevadas a cabo y las redes de emprendedores. Finalmente, se elaboran las conclusiones del estudio realizado.

\section{TRANSFORMACIÓN DE ESPACIOS GEOGRÁFICOS Y REDES}

Ante la tendencia creciente de la globalización y del turismo experiencial, la transformación de los espacios geográficos con patrimonio cultural se ha convertido en una opor- 
tunidad para el desarrollo económico y social. Manteniendo las características originales de dichos espacios: predominio de la pequeña empresa (Elizagarate, 2008), importancia de las relaciones locales, estructuras inter-empresariales basadas en la red, frente a las estructuras jerárquicas, y aprovechamiento de los bienes históricos y culturales (Amin y Robins, 1992), es posible desarrollar entornos únicos altamente valorados y demandados por los turistas (Sainz, 2015).

Las redes sociales son un elemento clave para coordinar la actividad económica (Granovetter, 1985). La literatura ha demostrado que facilitan el intercambio de recursos entre unidades, la innovación de productos y la creación de capital intelectual (Gabbay y Zuckerman, 1998; Nahapiet y Ghoshal, 1998), capital social (Adler y Kwon 2002), promoviendo la creatividad (Uzzi 1996; Uzzi y Spiro, 2005), la iniciativa empresarial y la formación de empresas de nueva creación (Walker, Kogut y Shan, 1997).

Por su carácter complejo, las redes sociales pueden articularse para desarrollar espacios únicos y no replicables, con una oferta de servicios y productos atractivos para el turismo personal y de negocios (Sorensen, 2007; Moulaert et al., 2005; Powell et al., 1996). El impacto de estas redes en la transformación de una zona comercial sería aún más relevante si se coordinan además con asociaciones de pequeños negocios y aprovechan herramientas de marketing social y de experiencias.

\section{TURISMO Y MARKETING DE EXPERIENCIAS}

Para los turistas antiguos las vacaciones eran un tiempo de ruptura con la vida cotidiana. Compraban paquetes que incluían transporte, alojamiento y diversión a un precio aceptable, eran bastante homogéneos y predecibles y la búsqueda de sol y playa era una constante en sus vacaciones. Sin embargo, los turistas actuales, consideran las vacaciones como una prolongación de su vida, algo común y normal. Estos turistas son "cazadores" de experiencias únicas, prefieren un turismo más individualizado y muestran un interés creciente por la tranquilidad, el contacto con la naturaleza y la cultura (Bigné, 2000; WTO, 2013; Valls et al., 2008; Cuenca y Prat, 2012).

Los datos del sector turístico muestran cambios en la forma de vacacionar. Se produce un mayor número de escapadas más cortas durante todo el año. En lugar de viajar una vez al año en temporada alta, casi un $40 \%$ de la demanda turística hace entre dos y tres viajes al año; y un 34\% entre cuatro y seis. La mayoría son vacaciones cortas, de tres a cuatro pernoctaciones, o escapadas de fin de semana (Valls et al., 2008). Los viajes son a la carta, individualizados y ajustados a los gustos del cliente.

Desde finales de los años noventa se percibe una mayor valoración de la calidad y la experiencia turística, del patrimonio cultural (Harris, 1990) y el paisaje en sentido integral. Las experiencias turísticas parecen identificarse cada vez más como el verdadero producto (Cuenca y Prat, 2012). Así, en la actualidad, adquiere cada vez más importancia el turismo experiencial o emocional.

El sector del Turismo Experiencial está en un proceso de transformación hacia unos productos y servicios centrados en la creación de experiencias y en la interactuación con el turista de forma más directa, humana y personalizada. Al integrar aspectos como la cultura y las tradiciones de las gentes del lugar, el turista se siente inmerso en su forma de vida, y 
sus sentimientos hacia el destino serán positivos (Molina et al. 2007; Troitiño, 1991). La experiencia es objeto esencial del viaje para una gran parte de los viajeros actuales, que sienten cada vez más la necesidad de experimentar un destino para optimizar su vivencia del mismo. Esta nueva tendencia, busca establecer una relación emocional entre el turista y el destino. Esta "economía de la experiencia" (Pine y Gilmore, 1998) lleva a las ciudades a convertirse en escenarios o "teatros" donde el turista se transforma en protagonista.

En la economía de la experiencia, la cultura se convierte en una materia prima esencial y el turismo cultural es un elemento cada vez más importante. Richards (2004) afirma que el turismo cultural ha pasado a convertirse en un producto de vivencia en el que la visita se juzga en función de todas las características del lugar, además de su valor cultural. En esta línea, los visitantes buscan cada vez más una experiencia total que incluya ocio, cultura, gastronomía e interacción social (De Rojas y Camarero, 2008; Rivera, 2013).

Un elemento clave y común de todas estas actividades es la creatividad y la innovación, que para desarrollarse requieren una red social (Uzzi y Spiro, 2005) de emprendedores que interactúen y colaboren. Por lo tanto, en este artículo se propone que el desarrollo y florecimiento económico de un área geográfica urbana única, que atrae turistas, requiere de una serie de actividades y estrategias de marketing experiencial que se generan porque existe una red colaborativa que incluye asociaciones de comerciantes, emprendedores y artistas.

\section{METODOLOGÍA Y DATOS DE LA INVESTIGACIÓN}

Para alcanzar los objetivos señalados en la investigación se han utilizado fuentes secundarias y primarias. En primer lugar, a partir de fuentes secundarias, revisamos las principales acepciones de redes y marcas de garantía y el perfil de este segmento de consumidores. También, estudiamos los principales cambios del sector turístico urbano hacia un marketing de experiencias y el perfil de este segmento de consumidores. Finalmente, recopilamos información secundaria sobre la historia del Barrio Las Letras, nuestro caso de estudio, y sobre la Asociación de Comerciantes del Barrio Las Letras.

Posteriormente, recurrimos a fuentes primarias de información, para así obtener de primera mano, datos sobre las características de la oferta cultural, comercial y turística del Barrio de Letras. Desarrollamos un modelo de entrevista a los emprendedores del Barrio de Las Letras con preguntas abiertas. Dichas entrevistas fueron relevantes para conocer las dinámicas y procesos sociales y cómo éstos impactan en el fenómeno que se está estudiando y se utilizaron para la elaboración de un cuestionario.

El cuestionario utilizado en esta investigación tiene dos secciones. La primera parte recoge información y datos sobre las características de los comerciantes y las acciones comerciales y llevadas a cabo. La segunda sección se basa en metodología de redes sociales (e.g. Cross y Parker 2004). El objetivo de esta sección es comprender como se articula la red de comerciantes y en qué medida dicha red impacta en la Asociación de emprendedores de Barrio de Las Letras, en sus acciones comerciales y también en toda la comunidad. En el Anexo se incorporan los cuestionarios utilizados.

El diseño y la aplicación de la encuesta personal a los emprendedores/comerciantes fue administrada en el período comprendido entre los meses de noviembre a diciembre de 2015 en el Barrio de Las Letras de Madrid. 
Determinamos un tamaño muestral de 187 unidades finales, fijando un nivel de confianza del $95 \%$ y al que le corresponde un nivel de significación del 5\%. De acuerdo con el tamaño de la muestra utilizado, de manera orientativa, el error máximo admisible cometido (para una estimación de proporciones) se eleva a un $+/-4 \%$ en condiciones de máxima incertidumbre $(\mathrm{p}=\mathrm{q}=50 \%)$.

El universo o población objetivo de esta fase de la investigación lo constituyen: comerciantes, servicios de restauración y de ocio de la zona divididos en varios grupos: comercios, hospedaje, servicios, hostelería, instituciones y cultura y turismo. En la tabla 1, que figura a continuación, se muestran los detalles técnicos del procedimiento de recolección de datos primarios.

Tabla 1

FICHA TÉCNICA DE LA INVESTIGACIÓN PRIMARIA

\begin{tabular}{|l|l|}
\hline Universo & Unidades comerciales del Barrio de las Letras \\
\hline Ámbito geográfico & Límites comerciales del Barrio de las Letras \\
\hline Muestra & $\begin{array}{l}187 \text { encuestas válidas (de los 301 comercios } \\
\text { asociados) }\end{array}$ \\
\hline Procedimiento de muestreo & Muestreo de conveniencia \\
\hline Error muestral & $\begin{array}{l}+/-4 \% \text { para un intervalo de confianza del 95\% } \\
\text { bajo la hipótesis de } \mathrm{p}=\mathrm{q}=0,5\end{array}$ \\
\hline Técnica de recogida de la información & $\begin{array}{l}\text { Encuesta personal administrada con } \\
\text { cuestionario estructurado }\end{array}$ \\
\hline Periodo de recogida de la información & Noviembre-Diciembre de 2015 \\
\hline Tratamiento de la información & Análisis univariable y bivariable descriptivo \\
\hline Procesamiento de la información & Dyane.SPSS 12.0. Excell \\
\hline
\end{tabular}

El análisis descriptivo de los datos arroja los siguientes resultados respecto al perfil de los negocios. El tamaño de los negocios es, en general, pequeño. La media de trabajadores son 7, si bien la mayor parte de los negocios tienen dos o tres empleados. Esta desviación $(16,76)$ es debida a que los alojamientos de hostelería y las empresas de restauración rondan la veintena empleados. El 68,11\% de los emprendedores encuestados son hombres, frente a un $31,89 \%$ de mujeres. Un $88,24 \%$ de los negocios son regentados por españoles.

El 50\% de las personas que han realizado el cuestionario son los propios emprendedores, siendo el otro $50 \%$, los empleados que dirigen el negocio. Un dato destacable es que el $65,78 \%$ de los negocios son gestionados por personas con estudios universitarios y un $23,53 \%$ poseen estudios secundarios.

\section{CASO DE INNOVACIÓN Y TRANSFORMACIÓN URBANA: BARRIO DE LAS LETRAS COMO DESTINO DE TURISMO EXPERIENCIAL}

Las nuevas tendencias en la industria han propiciado la aparición y el crecimiento de la oferta de turismo urbano de experiencias a las que dan respuesta los barrios "destino 
turístico", como es el caso del Barrio de Las Letras. La oferta cultural, comercial y de ocio del Barrio de las Letras supone una adaptación y remodelación de un casco histórico que oferta nuevas experiencias a los turistas con nuevas necesidades y, a todo ello, ha contribuido el asociacionismo y las redes de colaboración del pequeño comercio emprendedor de la zona, junto con instituciones culturales ubicadas en dicho barrio.

El Barrio de las Letras se encuentra ubicado en el centro de Madrid, es una referencia cultural y es miembro de la Confederación de Cascos Históricos (COCAHI). En esta zona de la capital vivió y murió Cervantes, nacieron los corrales de comedias, se encuentran algunos de los recursos patrimoniales destacables de la capital, siendo escenario de la mayor concentración de talento en la historia de la literatura universal.

En la actualidad, museos, galerías, restaurantes, pequeñas y originales tiendas, un mercado de abastos tradicional y una muy variada oferta de bares renuevan la imagen de la zona, no solo para seducir a los turistas sino también a los madrileños.

\section{RESULTADOS DEL ESTUDIO}

\subsection{El rol de una asociación de emprendedores}

La Asociación de Comerciantes, Autónomos y Pequeñas Empresas del Barrio de las Letras (Madrid) nace en octubre de 2005 con el propósito de establecer un plan de apoyo y promoción del Barrio de las Letras-BDLL por iniciativa de uno de los comerciantes del sector. Inicia su andadura con 30 socios y hoy ya la integran más de 300 asociados (Véase, la tabla 2, donde se puede observar la evolución de los asociados por año).

Entre todos los asociados se lleva a cabo el proyecto de dinamización comercial, y de regeneración del barrio, que se constituye como un referente en el corazón de Madrid, ofreciendo un "microdestino" turístico con actividades que permiten al viajero entrar en contacto con el estilo de vida local y con la cultura de los residentes.

Los objetivos de la Asociación son claros:

- Conseguir un comercio cercano y competitivo, donde la calidad, la profesionalidad y el servicio (atención al cliente) sean las señas de identidad, dentro de una amplia oferta comercial.

- Proteger y poner en valor el comercio tradicional, así como fomentar el asentamiento de nuevas ideas emprendedoras, que enriquezcan el tejido socioeconómico del Barrio.

- Potenciar y consolidar el asociacionismo, que genera riqueza y fomenta una cultura de cooperación.

- Articular las bases necesarias para la creación y consolidación de un Área Comercial Urbana, dotándola de una estrategia común, así como de una imagen corporativa que refuerce la identidad dentro y fuera del Barrio de las Letras, mostrando la oferta que se genera en el mismo. 
Tabla 2

EVOLUCIÓN DE ASOCIADOS POR AÑO

\begin{tabular}{|c|c|}
\hline Año & Total Asociados \\
\hline 2005 & 63 \\
\hline 2006 & 135 \\
\hline 2007 & 161 \\
\hline 2008 & 192 \\
\hline 2009 & 209 \\
\hline 2010 & 221 \\
\hline 2011 & 237 \\
\hline 2012 & 265 \\
\hline 2013 & 272 \\
\hline 2014 & 295 \\
\hline 2015 & 317 \\
\hline 2016 & 333 \\
\hline
\end{tabular}

Fuente: Asociación de Comerciantes. Barrio de Las Letras (2016).

Los dos primeros objetivos, en cuanto al comercio tradicional y los nuevos emprendedores como sello de identidad se consiguen con la amplia oferta comercial de pequeñas tiendas, restaurantes originales, galerías de arte y otros negocios, siempre a pequeña escala, evitando franquicias comerciales y marcas "main street". Todo ello da lugar a un "carácter de autenticidad" del barrio con una oferta comercial muy variada y original.

Otra acción, que se lleva a cabo de manera periódica para lograr los objetivos anteriormente mencionados, es mantener continuas reuniones de coordinación con la Policía Nacional y Municipal para mantener el barrio seguro. Adicionalmente, se trabaja en la formación continua de los asociados. También se ha establecido una importante colaboración con las grandes instituciones del Barrio de las Letras: Real Academia de la Historia, Casa-Museo Lope de Vega, Teatro Español, Caixa Forum, Monasterio de las Trinitarias, Medialab, Ateneo de Madrid, etc. Dichas instituciones son un referente a nivel de recurso turístico cultural en Madrid, lo que otorga al Barrio un aire literario, al que refiere su nombre, y que es base de la experiencia ofrecida a los turistas.

Finalmente, desde la Asociación se han fijado tres ejes de trabajo que conforman las señas de identidad del BDLL: Comercio, Cultura y Turismo.

- Comercio: El BDLL ofrece una rica variedad de establecimientos donde los más diversos productos y servicios se ofertan. Desde las más genuinas librerías de Madrid, a las galerías de arte más vanguardista, pasando por tiendas de moda, antigüedades, decoración, etc.

- Cultura: Está presente en cada piedra del Barrio, decenas de placas conmemorativas nos recuerdan los hechos, los personajes, las historias que hacen que el BDLL sea un de los barrios más importantes de Madrid. 
- Turismo: Es uno de los principales motores del Barrio. Cada año millones de personas pasean por las calles del BDLL, disfrutan de su rica oferta de ocio, gastronomía y se alojan en alguno de los más de treinta hoteles que jalonan el barrio.

\subsection{Oferta de servicios y marketing experiencial}

Respecto a la oferta comercial, esta se centra principalmente en varios tipos de negocios, tal como la asociación los tiene catalogados por tipo de actividad (la tabla 3 recoge esta información). De esta gran oferta de servicios el perfil de los encuestados es una muestra representativa de toda la población comercial del barrio.

Tabla 3

OFERTA COMERCIAL DEL BARRIO DE LAS LETRAS SEGÚN ACTIVIDAD COMERCIAL

\begin{tabular}{|l|ll|}
\hline \multicolumn{1}{|l|}{ Actividad principal } & \\
\hline Comercios & Alimentación & Salud \\
& Antigüedades & Informática/Web \\
& Arte & Instrumentos musicales \\
& Belleza & Joyerías y bisuterías \\
& Comunicaciones & Librerías y papelerías \\
& Flores y plantas & Moda. Textil y complementos \\
& Fotografía & Tiempo libre \\
& Hogar y Decoración & Reparación y venta de motocicletas \\
& Impresión y artes gráficas & Otras actividades comerciales \\
\hline Hospedaje & Hoteles & Pensiones \\
& Hostales & \\
\hline Servicios & Artes escénicas & Entidades Financieras \\
& Asesoría y consultoría & Gimnasio/spa \\
& Belleza & Inmobiliarias \\
& Enseñanza & Salud. Centros sanitarios \\
& Comunicaciones & Tiempo libre \\
& Clínica Veterinaria & Otros servicios \\
& Editorial & \\
\hline Hostelería & Bares y Restaurantes & Ocio y Espectáculos \\
\hline Instituciones & Ateneo & Caixa Forum Madrid \\
& Iglesia Jesús de Medinaceli & Casa Museo Lope de Vega \\
& MediaLab & Teatro Español \\
& Monasterio Trinitarias & \\
\hline & Ateneo & Caixa Forum Madrid \\
& M.N.C.A. Reina Sofía & Museo Nacional del Prado \\
\hline
\end{tabular}

Fuente: elaboración propia basada en datos provistos por la Asociación de Comerciantes. Barrio de Las Letras (2016) y www.barrioletras.com 
La actividad predominante es el comercio y la hostelería restauración, por ello el 50\% de la muestra aproximadamente son comerciantes (95 encuestados) y el 35\% se dedica a la hostelería y restauración (67 encuestados). En concreto, de los 95 comercios analizados, un $21,05 \%$ se dedican a la moda, un $15,79 \%$ al arte, un $9,47 \%$ son tiendas de antigüedades y un $8,42 \%$ tiendas de alimentación, un 6,32 son librerías y también un 6,32\% son tiendas de decoración (véase figura 1).

\section{Figura 1 \\ ACTIVIDAD PRINCIPAL DE LOS \\ COMERCIOS DEL BARRIO DE LAS LETRAS}

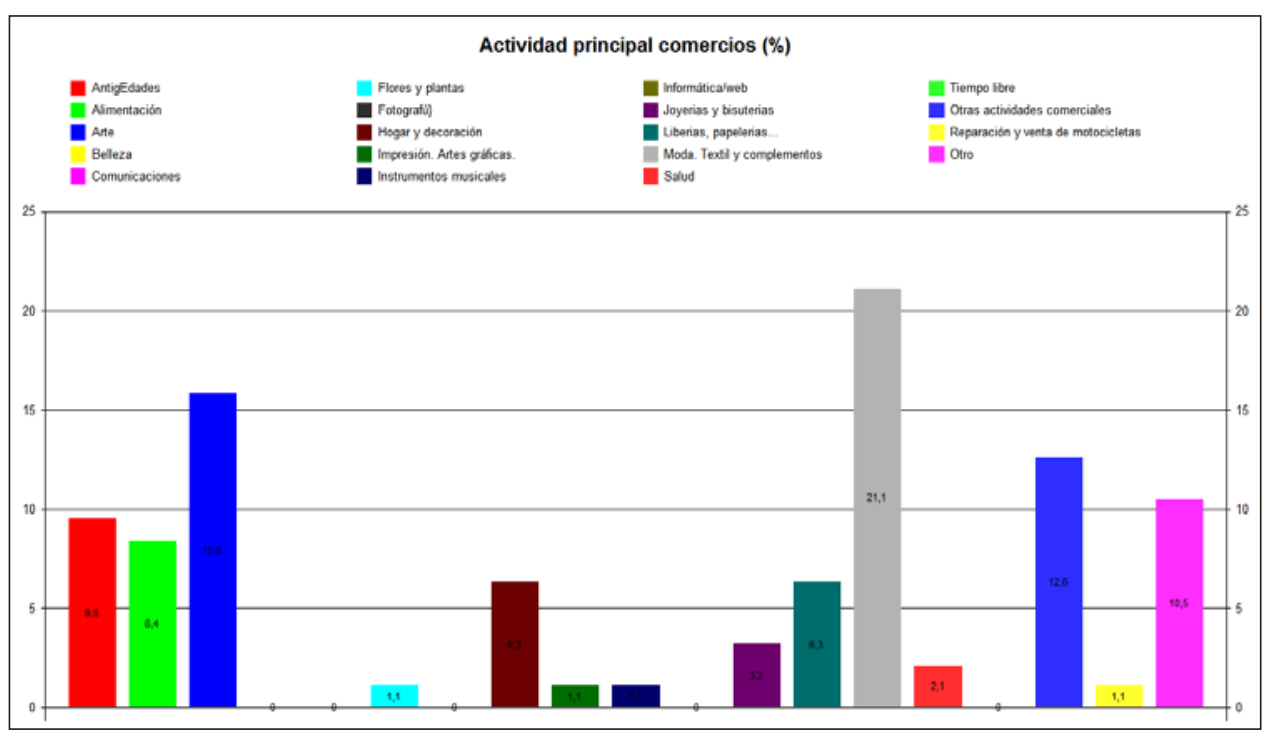

Fuente: elaboración propia (2016).

Respecto al hospedaje (9 encuestados), el 66,67\% son hoteles, un 11,11 hostales y un $22 \%$ Pensiones. Por otra parte, los negocios cuya actividad principal son servicios suponen un $10 \%$ de los encuestados y son principalmente inmobiliarias $(15,79 \%)$, salones de belleza $(10,53 \%)$ y otros servicios $(52,63 \%)$.

Para dinamizar las tres áreas de trabajo de la Asociación, mencionadas previamente (Comercio, Cultura y Turismo), desde la Asociación se promueven múltiples actividades y campañas de comunicación que muestren a los vecinos, a los visitantes y a los turistas la rica oferta del Barrio de Las Letras.

No se ha de olvidar que el turista experiencial viaja normalmente de forma independiente y usa internet y otros medios de comunicación on-line para informarse de las actividades en el destino. Ante este panorama, los destinos turísticos han de considerar el uso de las plataformas de redes sociales y el ciberespacio como su principal herramienta de comunicación con el nuevo nicho de mercado turístico que busca nuevas experiencias, utilizando Webs, redes sociales y blogs, como instrumentos de promoción. Mediante el uso 
de descripciones en texto, imágenes, mapas, videos y comentarios de usuarios e influenciadores es posible sentir o adelantar la experiencia del viaje (Serra 2011), algo de suma importancia para los destinos y productos que centran su oferta en el turismo experiencial, como es el caso de los eventos organizados por el Barrio de las Letras. El "boca oreja", tan importante en el marketing turístico, se ve ahora potenciado por los miles de contactos que un usuario activo en internet puede generar mediante blogs, correo electrónico, su cuenta de Facebook o Twitter, etc.

Una de las acciones de marketing más importantes ha sido la creación de la marca o etiqueta Barrio de Las Letras, asociada a un destino turístico dentro de un barrio céntrico de Madrid. Las estrategias de creación de sellos o labels tienen objetivos muy diversos. Desde la perspectiva de la oferta, como objetivos comunes genéricos, cabe destacar que se trata de un instrumento de protección, promoción y comercialización. Respecto a la demanda, se trata de una importante fuente de información y significado para el público objetivo. Como medio de información el turista utiliza el Sello para identificar el origen del servicio/destino y conocer algunas particularidades sobre sus características y nivel de calidad. Mientras que, como fuente de significado, el Sello permite incorporar y reforzar algunas asociaciones vinculadas al servicio, permitiéndole identificar nuevos beneficios, mejorar su nivel de conocimiento, y sobre todo, construir y reforzar la imagen que se crea en su mente sobre el mismo (Jiménez at al., 2006).

Van Trijp y otros (1995) argumentan que los labels de calidad ayudan a los consumidores imperfectamente informados en su proceso de decisión de compra, porque estructuran su entorno informativo. Mientras, autores como Papadopulos y Heslop (1993) describen la imagen de los labels como la de los productos, que en este caso sería un destino turístico como es el Barrio de las Letras en la ciudad de Madrid.

Según el estudio realizado, las principales acciones comerciales de los últimos años son las que se recogen en la Tabla 4. De las acciones comerciales enumeradas en esta tabla, algunas de ellas son mayormente utilizadas por los comerciantes de la zona, creando un ambiente de experiencias vividas que los turistas valoran especialmente.

\section{Tabla 4}

\section{PRINCIPALES ACCIONES COMERCIALES LLEVADAS A CABO POR LA ASOCIACIÓN BARRIO DE LAS LETRAS}

\begin{tabular}{|l|l|l|l|l|l|l|l|}
\hline \multicolumn{1}{|c|}{ Acción Comercial } & $\mathbf{2 0 0 9}$ & $\mathbf{2 0 1 0}$ & $\mathbf{2 0 1 1}$ & $\mathbf{2 0 1 2}$ & $\mathbf{2 0 1 3}$ & $\mathbf{2 0 1 4}$ & $\mathbf{2 0 1 5}$ \\
\hline DecorAcción & & & & & & & \\
\hline Mercado de las Ranas & & & & & & & \\
\hline La noche de los libros & & & & & & & \\
\hline Fidelización turística & & & & & & & \\
\hline Black Friday & & & & & & & \\
\hline Nos vamos de BARTES & & & & & & & \\
\hline $\begin{array}{l}\text { Gastro Bacchus: de tapa Madrid de } \\
\text { los vinos }\end{array}$ & & & & & & & \\
\hline
\end{tabular}

Fuente: Asociación de Comerciantes de BDLL. 
La Tabla 5, recoge las principales acciones comerciales y la definición de las mismas en referencia a oferta de marketing experiencial bajo el paraguas de la marca "Barrio de Las Letras".

\section{Tabla 5 \\ DEFINICIÓN E IMAGEN DE MARCA DE LAS PRINCIPALES ACCIONES COMERCIALES LLEVADAS A CABO POR LA ASOCIACIÓN BARRIO DE LAS LETRAS Y NÚMERO DE PARTICIPANTES}

\begin{tabular}{|c|c|}
\hline Merc & $\begin{array}{l}\text { - Número de encuestados que han participado en dicha acción comercial: } 116 \text { (62\%) } \\
\text { - Los establecimientos del barrio sacan a la calle su oferta comercial, cultural, gas- } \\
\text { tronómica y artística los primeros sábados de cada mes. Además de un mercado } \\
\text { abierto los establecimientos de hostelería ofrecen numerosos atractivos para apor- } \\
\text { tar una oferta integral a todos los visitantes } \\
\text { - Su objetivo es impulsar el desarrollo económico del Barrio y potenciar la ciudad } \\
\text { de Madrid como referente turístico nacional e internacional. } \\
\text { - Impulsado por la Asociación de Comerciantes del Barrio de las Letras en cola- } \\
\text { boración con el Ayuntamiento de Madrid, y con el apoyo del sector hotelero y } \\
\text { diversas instituciones y entidades públicas y privadas del Barrio. } \\
\text { - Emulando otros mercados mundialmente conocidos, como el Portobello Road o } \\
\text { Camden Town, en Londres; el Soho Antiques Fair de Nueva York; o el parisino } \\
\text { mercado de las pulgas de Saint-Ouen. }\end{array}$ \\
\hline & $\begin{array}{l}\text { - Número de encuestados que han participado en dicha acción comercial: } 112 \text { (59\%) } \\
\text { - Organizado por la Asociación de Comerciantes, la revista Nuevo Estilo y el Ayun- } \\
\text { tamiento de Madrid, donde el diseño y el interiorismo toman las calles. } \\
\text { - Actividades: Mercadillo de Anticuarios (más de 60); Mercadillo Pop Up cita jóve- } \\
\text { nes artesanos y artistas; y foodtrucks, gastronomía, decoración y talleres gratuitos. }\end{array}$ \\
\hline & $\begin{array}{l}\text { - Número de encuestados que han participado en dicha acción comercial: } 65 \\
(34,7 \%) \\
\text { - Barrio de las Letras se ha adaptado la fórmula de Black Friday americano. } \\
\text { - Descuentos que oscilan entre el } 10 \text { y el 50\%, tanto en sus locales como en los } \\
\text { casos de poseer sitio web. Como se pudo comprobar en los diversos medios de } \\
\text { comunicación, la aceptación cada vez mayor, con mayor afluencia de visitantes a } \\
\text { las calles del Barrio de las Letras }\end{array}$ \\
\hline$E M$ & $\begin{array}{l}\text { - Número de encuestados que han participado en dicha acción comercial: } 25 \\
(13,2 \%) \\
\text { - La Noche de los Libros es una actividad cultural madrileña realizada cada } 23 \text { de } \\
\text { abril, coincidiendo con el Día Internacional del Libro } \\
\text { - En el Barrio de las Letras se abren librerías, bibliotecas y entornos literarios hasta } \\
\text { media noche y se organizan actividades culturales en torno a la literatura } \\
\text { - En } 2015 \text { junto a la Escuela de Esgrima Ateneo / Barrio de las Letras } \\
\text { - Representaciones teatrales y librerías con programas de actividades y apertura } \\
\text { hasta media noche, hacen un } 10 \% \text { de descuento en libros para promover la lectura }\end{array}$ \\
\hline
\end{tabular}

Fuente: Asociación de Comerciantes de BDLL. 
A nivel comercial y de afluencia de público las acciones más exitosas son las que abarcan a mayor número y tipo de negocios. Los asociados realizan varias acciones simultáneamente, aunque, sin duda El Mercado de las Ranas y DecorAcción son los dos eventos de mayor participación y también de mayor repercusión en los medios y, por ello, de mayor recepción de turistas. Por ejemplo, la asociación estima que en DecorAcción participaron 220.000 asistentes en 2016 y 200.000 en 2015.

Los datos de la investigación revelan que El Mercado de las Ranas es un evento que ha evolucionado de forma muy positiva desde su inicio, como recoge la Figura 2.

El 62\% de los encuestados (116 empresarios) han participado en alguna o varias de las convocatorias de dicho Mercado. La implicación de esos 116 empresarios ha manifestado un crecimiento continuado desde el comienzo de dicho evento, que se celebra de forma mensual, ya que en 2012 participó el 58,52\% (68 asociados) de esos 116 empresarios y en 2015 el 94,93\% (110 asociados de los 116 emprendedores que han participado en alguna de las convocatorias).

\section{Figura 2 \\ IMPLICACIÓN DE LOS ASOCIADOS EN EL MERCADO DE LAS RANAS}

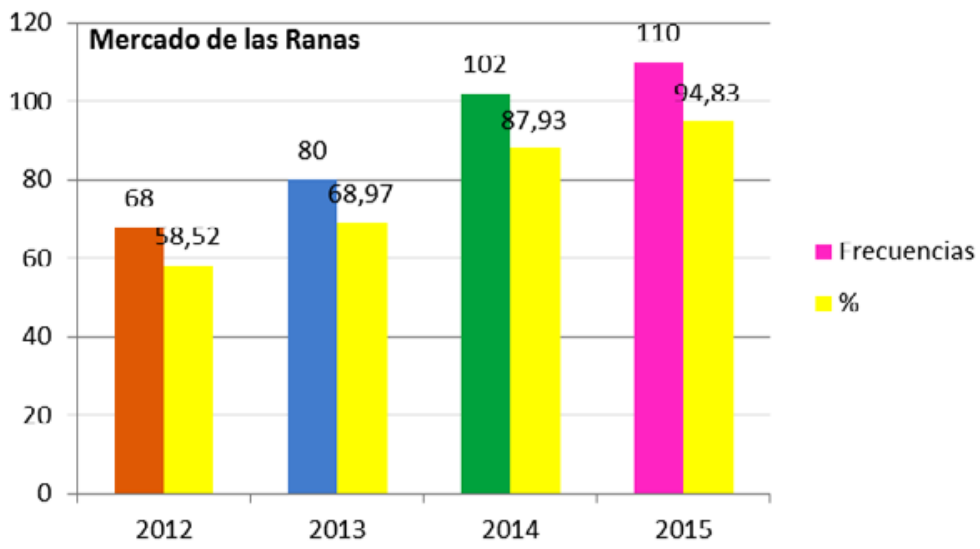

Fuente: elaboración propia (2016).

El segundo gran evento cultural, comercial y gastronómico es DecorAccion, con una participación de un 59\% (112 de los 189 emprendedores analizados) de los encuestados que han participado en alguna o varias de las convocatorias de dicho evento anual (véase Figura 3). Igualmente que en la acción comercial del Mercado de las Ranas, la participación en DecorAcción ha ido en aumento a lo largo de los años, siendo de un 51,79\% de los 112 participantes en 2010 y pasando a 93,75\% de los 112 participantes en 2015, alcanzando casi el doble de participación frente a la primera convocatoria.

Por otra parte, este evento es el de mayor repercusión en medios de comunicación de tendencias, moda, decoración y turismo, lo que supone un posicionamiento del Barrio de Las Letras como marca turística de micro destino para muchos pequeños nichos de mercado. 


\section{Figura 3 \\ IMPLICACIÓN DE LOS ASOCIADOS EN EL EVENTO DECORACCION}

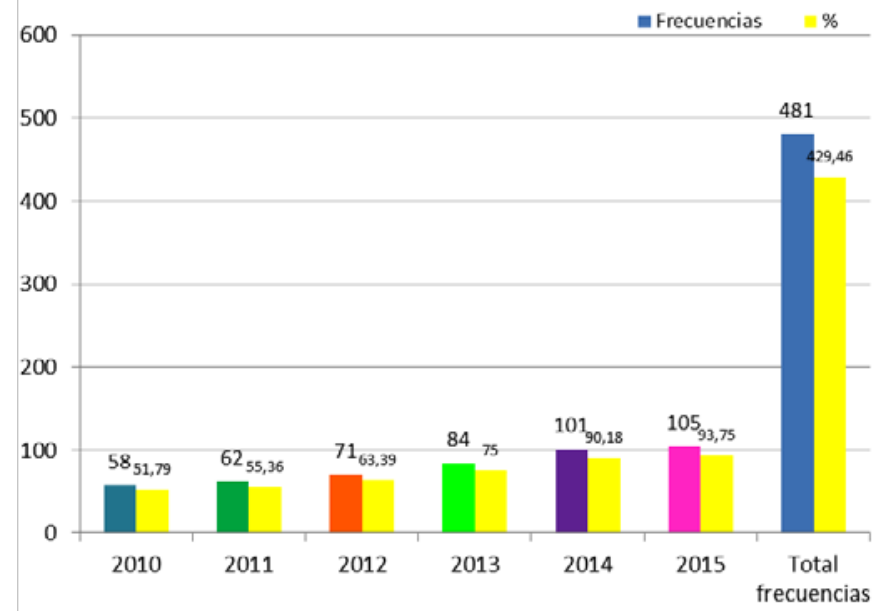

Fuente: elaboración propia (2016).

Por último es interesante destacar las acciones culturales llevadas a cabo en La Noche de los Libros, donde el barrio refleja la influencia de su denominación como "lugar de referencias literarias". En este evento, además de las librerías y otros comerciantes, se implican otras instituciones como la Real Academia de la Historia, Casa-Museo Lope de Vega, Teatro Español, Caixa Forum, Monasterio de las Trinitarias, Medialab, Ateneo de Madrid, etc.

\subsection{Asociacionismo y redes}

La Asociación de Comerciantes del Barrio de las Letras aglutina a pequeños emprendedores y pequeños comercios del área. Las redes entre estos emprendedores permiten coordinarse rápidamente y generar las actividades antes descritas y que tienen como objetivo adaptarse a las necesidades de los turistas urbanos, con un tinte único y diferenciador.

La figura 4 que se representa a continuación, muestra la red de colaboración entre los pequeños negocios que forman parte de la Asociación de Comerciantes del Barrio de las Letras. Los vínculos se construyen a partir de las respuestas de los mismos comerciantes y emprendedores a las preguntas que figuran en el Anexo 1, parte II.

Es interesante observar que el nivel de interrelación y colaboración entre los emprendedores es alto. De los 364 nodos (aquí los nodos representan a todos los negocios y establecimientos mencionados por los encuestados) que forman parte de la red, 321 $(88,2 \%)$ forman parte del componente principal (es decir la subred que abarca al mayor número de nodos conectados entre sí). Este resultado demuestra que la cooperación es muy importante para que los emprendedores del Barrio de las Letras puedan desempeñarse adecuadamente y para que brinden servicios de calidad. 


\section{Figura 4 \\ RED DE COOPERACIÓN Y COLABORACIÓN ENTRE COMERCIOS Y EMPRENDIMIENTOS DE BARRIO DE LAS LETRAS}

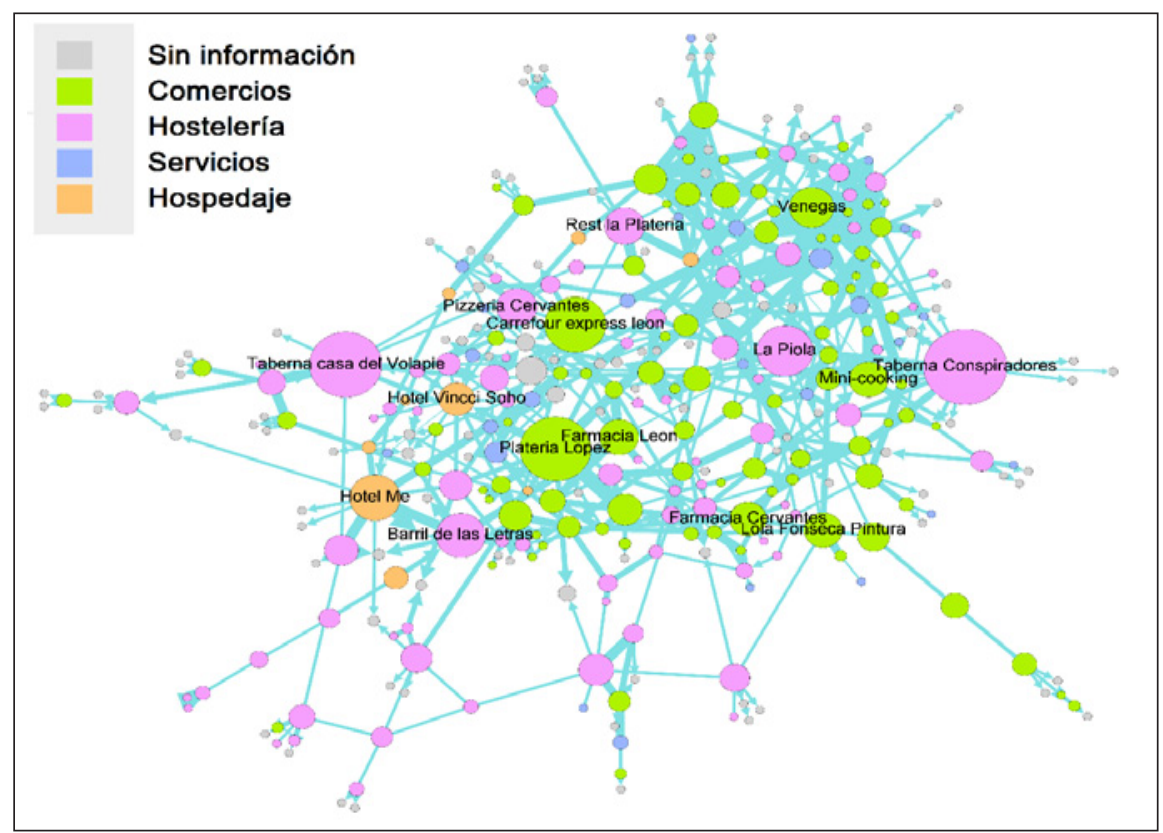

Sin embargo, la red tiene una densidad (que es la cantidad de vínculos respecto de todos los vínculos posibles en la red) baja, de 1,1\%. El diámetro (o la cantidad de pasos que existen entre los nodos más distantes de la red) es 13 y el grado (o la cantidad promedio de vínculos de un emprendedor) es de 4. Por lo tanto, estamos ante una red que integra a un gran porcentaje de los emprendedores del Barrio de las Letras (88\%), pero que es bastante dispersa. Esta dispersión, permitiría la existencia de agujeros estructurales (Burt, 1995) necesarios la generación de oportunidades, información diversa y nuevos emprendimientos.

El color de los nodos está asociado al sector. Los restaurantes y tabernas son los más activos relacionadores de la red y posteriormente algunos hoteles y comercios como supermercados, farmacias y un negocio de platería. El ancho de los vínculos representa la fortaleza de las relaciones entre los comercios.

El tamaño de los nodos de la figura 4 muestra a los negocios más activos a la hora colaborar y pedir colaboración y que son muy importantes para la creación de un ecosistema único. Entre los más activos y relacionados se encuentran Taberna los Conspiradores, Taberna Casa del Volapié, Hotel Me o la Platería López. Si estos emprendedores cerraran sus comercios y se fueran, la red se vería damnificada y se fragmentaría. Por ello, es importante valorar el capital social que estos emprendedores aportan. Ellos tienen más información y conocimiento que el resto, mantienen al grupo integrado y son fundamentales para que la información y las ideas se difundan y conversen entre los emprendedores del Barrio. 
La figura que se muestra a continuación (figura 5), es similar a la anterior, pero con la única diferencia que el color de los nodos muestran quienes son asociados (azul) o no lo son (anaranjado).

\section{Figura 5 \\ RED DE COOPERACIÓN Y COLABORACIÓN ENTRE COMERCIOS}

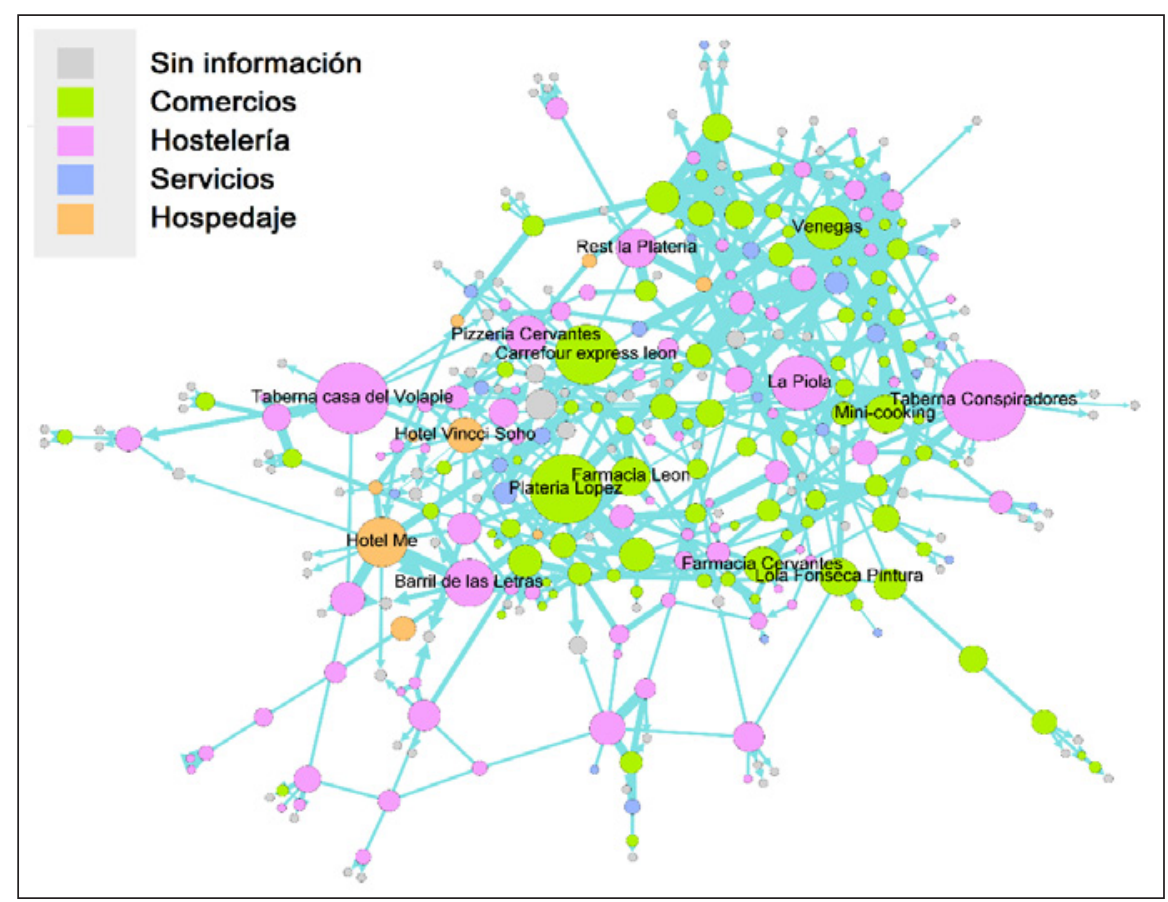

En la Tabla 6 se listan los comercios y emprendedores más centrales del Barrio de las Letras. Se utilizan dos medidas de centralidad diferentes.

La centralidad de intermediación y la centralidad de grado. La idea fundamental del concepto de Centralidad de Intermediación (betweenness) es que un actor es central si está entre otros actores en sus distancias geodésicas. La distancia geodésica es el camino más corto entre un par de nodos. Esto implica que para tener un índice de Centralidad de Intermediación alto, el actor debe estar entre muchos otros actores en sus respectivas distancias geodésicas (Wasserman y Faust, 1994). La centralidad de intermediación muestra en qué medida un actor controla los flujos de información dentro de la red. Por otra parte, se utiliza la centralidad de grado local (degree) para medir que parte de la actividad de la red pasa por un nodo. La centralidad de grado se mide a partir del número de conexiones directas que tiene un nodo con el resto de los nodos de la red. Un actor con alta centralidad de grado está en contacto directo o es adyacente a otros muchos actores. Esta medida se calcula sumando la cantidad de vínculos de cada nodo. 


\section{Tabla 6 \\ RANKING DE CENTRALIDAD DE COMERCIOS Y EMPRENDIMIENTOS EN BARRIO DE LAS LETRAS}

\begin{tabular}{|c|c|c|c|c|c|c|}
\hline Ranking & $\begin{array}{l}\text { Centralidad de } \\
\text { intermediación }\end{array}$ & Nombre & Sector & \begin{tabular}{|c|} 
Centralidad \\
de Grado
\end{tabular} & Nombre & Sector \\
\hline 1 & 0.11 & Taberna Conspiradores & 4 & 19 & \begin{tabular}{|l|} 
Taberna \\
Conspiradores
\end{tabular} & 4 \\
\hline 2 & 0.10 & Taberna Casa Del Volapie & 4 & 19 & Venegas & 1 \\
\hline 3 & 0.09 & Platería López & 1 & 19 & Érase Una Vez. .. & 4 \\
\hline 4 & 0.08 & Carredour Express Leon & 1 & 17 & Valyrium & 1 \\
\hline 5 & 0.07 & Restaurante A'cañada & 4 & 13 & \begin{tabular}{|l} 
Taberna Casa Del \\
Volapie
\end{tabular} & 4 \\
\hline 6 & 0.06 & Hotel Me & 2 & 13 & Platería López & 1 \\
\hline 7 & 0.06 & El Barril De Las Letras & 4 & 13 & Hotel Me & 2 \\
\hline 8 & 0.05 & Venegas & 1 & 13 & Ginger \& Velvet & 1 \\
\hline 9 & 0.05 & Pizzería Cervantes & 4 & 13 & Miseria & 1 \\
\hline 10 & 0.05 & Rest La Platería & 4 & 12 & \begin{tabular}{|l} 
Hotel Vincci \\
Soho
\end{tabular} & 2 \\
\hline 11 & 0.05 & Farmacia León & 1 & 12 & Mazarias & 1 \\
\hline 12 & 0.05 & Mini-Cooking & 1 & 12 & Motteau & 4 \\
\hline 13 & 0.04 & $\begin{array}{l}\text { Lola Fonseca Pintura En } \\
\text { Seda }\end{array}$ & 1 & 12 & Il Guacciaro & 1 \\
\hline 14 & 0.04 & Farmacia Cervantes & 1 & 11 & Losada & 1 \\
\hline 15 & 0.04 & Hotel Vincci Soho & 2 & 11 & $\begin{array}{l}\text { Droguería. Pefu } \\
\text { Castillo }\end{array}$ & 1 \\
\hline 16 & 0.04 & Restaurante Matute & 4 & 10 & $\begin{array}{l}\text { Carredour } \\
\text { Express Leon } \\
\end{array}$ & 1 \\
\hline 17 & 0.04 & Olarra & 1 & 10 & $\begin{array}{l}\text { Pizzería } \\
\text { Cervantes }\end{array}$ & 4 \\
\hline 18 & 0.04 & Naturbier & 4 & 10 & Mini-Cooking & 1 \\
\hline 19 & 0.04 & Librería Del Prado & 1 & 10 & Olarra & 1 \\
\hline 20 & 0.04 & Ginger \& Velvet & 1 & 10 & Bodegas Trigo & 1 \\
\hline
\end{tabular}

Es interesante ver que los comercios que forman parte de la Asociación del Barrio de las Letras, no solo establecen lazos de colaboración con otros miembros sino que también lo hacen con comercios u organizaciones que no forman parte de la asociación e incluso del propio barrio. 


\section{CONCLUSIONES E IMPLICACIONES}

El desarrollo y florecimiento económico de un área geográfica urbana única, que atrae turistas, requiere de una serie de actividades y estrategias de marketing experiencial que se generan porque existe una red colaborativa que incluye asociaciones de comerciantes, emprendedores y artistas.

Las nuevas tendencias en la industria han propiciado la aparición y el crecimiento de la oferta de turismo urbano de experiencias a las que dan respuesta los barrios "destino turístico", como es el caso del Barrio de Las Letras. La oferta cultural, comercial y de ocio del Barrio de las Letras supone una adaptación y remodelación de un casco histórico que oferta nuevas experiencias a los turistas con nuevas necesidades y a todo ello ha contribuido el asociacionismo y las redes de colaboración del pequeño comercio emprendedor de la zona, junto con instituciones culturales ubicadas en dicho barrio.

Los sectores preponderantes en el Barrio de Las Letras son los pequeños comercios, empresas de servicios y los establecimientos de restauración y alojamiento de tipo mediano. La actividad predominante es el comercio y la hostelería restauración, por ello el $50 \%$ de la muestra aproximadamente son comerciantes (ante todo de moda, arte y antigüedades y librerías) y el $35 \%$ se dedica a la hostelería y restauración.

El perfil de los emprendedores se podía definir como hombres con estudios universitarios.

Respecto a las actividades comerciales es interesante el incremento sostenido en lo que se refiere a actividades de la asociación y también el número de emprendedores que forman parte de los mismos. Los asociados realizan varias acciones simultáneamente, aunque, sin duda El Mercado de las Ranas y DecorAcción son los dos eventos de mayor participación y también de mayor repercusión en los medios y, por ello, de mayor recepción de turistas

El estudio revela que el desarrollo de espacios urbanos que tienen como objetivo ofrecer turismo de experiencias generando una ecología de redes y actividades que se torna compleja y con varios niveles. Un nivel son las redes individuales entre los emprendedores, que se estudiaran con posterioridad en las siguientes fases del proyecto. Adicionalmente, existen las actividades organizadas por la asociación que promueven redes entre quienes participan en las mismas. Finalmente, la asociación de emprendedores de Barrio de las Letras es una red en sí misma.

Los primeros datos sobre redes de colaboración muestran las conductas relacionales de los emprendedores de barrio de las letras y como éstas impactan en sus acciones. Se observa que el nivel de interrelación y colaboración entre los emprendedores es alto y la cooperación es muy importante para que los emprendedores del Barrio de las Letras puedan desempeñarse adecuadamente y para que brinden servicios de calidad.

La investigación futura intentará responder a preguntas como: ¿los actores más relacionados son también los más proclives a participar en las actividades de la Asociación y por lo tanto más comprometidos con la construcción de la marca Barrio de las Letras? ¿Quiénes más relaciones gestionen obtienen mejores resultados? ¿Cómo evoluciona la red de colaboración entre los emprendedores del Barrio de las Letras? ¿Cómo afecta la marca Barrio de las Letras en la comercialización turística internacional? 


\section{BIBLIOGRAFÍA}

ADLER, P. y KWON, S (2002): «Social Capital: Prospects for a new concept», The Academy Management Review, $\mathrm{n}^{\circ} 27$, pp. 17-40.

AMIN, A. y ROBINS, K. (1992), «The re-emergence of regional economies?, The mythical geography of flexible accumulation», Environment and Planning D: Society and Space, vol. 8, pp. 7-34.

BIGNE ALCAÑIZ, E. (2000): Marketing de destinos turísticos: análisis y estrategias de desarrollo, Esic, Madrid.

BURT, R. (1995): Structural Holes. The Social Structure of Competition, Harvard University Press.

CROSS, R. y PARKER, A. (2004): The hidden power of social networks. Harvard Business School Press.

CUENCA, M. y PRAT, A. (2012): «Ocio experiencial: antecedentes y características», Arbor. Ciencia, Pensamiento y Cultura, vol. 188, no 756, pp. 265-281.

DE ROJAS, C. y CAMARERO, C. (2008): «Visitors experience, mood and satisfaction in A heritage context: Evidence from an interpretation center», Tourism Management, $\mathrm{n}^{\circ} 29$, pp. 525-537.

ELIZAGARATE, V. (2008): Marketing de ciudades: estrategias para el desarrollo de ciudades atractivas y competitivas en un mundo global, Pirámide, Madrid.

GABBAY, S.M. y ZUCKERMAN, E.M. (1998): «Social capital and opportunity in corporate R\&D: The contingent effect of contact density on mobility expectations», Social Science Research.vol. 27 (2), pp. 189-217.

GRANOVETTER, M.S. (1985): «Economic action and social structure: the problem of embeddedness», The American Journal of Sociology, $\mathrm{n}^{\circ} 91$ (3), pp. 487-510.

HARRIS, S.G. (1994): «Organizational culture and individual sensemaking: a schemabased perspective», Organization Science, vol. 5, no 3, pp. 309-321.

JIMÉNEZ-ZARCO A.I., MARTÍNEZ-RUÍZ, P., y GONZÁLEZ-BENITO, O. (2006): «Performance Measurement System Integration into New Product Innovation: A Literature Review and Conceptual Framework», Academy of Marketing Science Review, vol. 2006, no. 9, pp. 1-16.

MOLINA, A., MARTÍN CONSUEGRA, D., ESTEBAN, A. y DÍAZ, E. (2007): «Segmentación de la demanda turística: Un análisis aplicado a un destino cultural», Revista de análisis turístico, $\mathrm{n}^{\circ}$ 4. pp. 36-48.

MOULAERT F., FLAVIA MARTINELLI, F., SWYNGEDOUW, E. y GONZÁLEZ, S. (2005): «Towards Alternative Model(s) of Local Innovation», Urban Studies, vol. 42, n 11 , pp. 1969-1990.

NAHAPIET, J. y GHOSHAL, S. (1998): «Social capital, intellectual capital, and the organizational advantage », The Academy of Management Review, vol. 23, n 2, pp. 242-266.

PAPADOPOULOS, N. y HESLOP, L.A. (1993): Product-Country Images: Importance and Role in International Marketing, International Business Press, New York.

PINE, B.J. y GILMORE, J.H (1999): The experience economy: Work is Theater\& Every Business a Stage, Harvard Business School Press, Boston. 
POWELL, W.W.; KOPUT, K.W. y SMITH-DOERR, L. (1996). «Interorganizational collaboration and the locus of innovation: Networks of learning in biotechnology», Administrative Science Quarterly, $\mathrm{n}^{\circ}$ 41, pp. 116-145. 11.

RICHARDS (2004): «¿Nuevos caminos para el turismo cultural?», Interacció. Centro de Estudios Culturales CERC, pp. 1-15. Disponible en www.diba.es/cerc/arxinterac04/ arcem1/richards/dipbarcelona

RIVERA MATEOS, M. (2013): «El Turismo Experiencial Como Forma De Turismo Responsable E Intercultural», pag. 199-217. Jornadas sobre Investigación e Innovación para la Interculturalidad, Córdoba 2013.

SAINZ ALVAREZ, M. (2015): «La ciudad en la economía de la experiencia: diseño, gestión y rol de los ciudadanos. Reflexiones y propuestas desde Bilbao». Working paper presentado en International Conference on Regional Science. Oviedo. Disponible en http://www.reunionesdeestudiosregionales.org/Oviedo2013/htdocs/pdf/p658.pdf

SERRA CANTALLOPS, A. (2011): Marketing Turístico, Editorial ESIC-Pirámide, Madrid.

SØRENSEN, F. (2007): «The Geographies of Social Networks and Innovation in Tourism», Tourism Geographies: An International Journal of Tourism Space, Place and Environment, vol. 9, n 1, pp. 22-48.

TERÁN ALVAREZ, M. DE (1966): «La ciudad como forma de ocupación del suelo y organización del espacio», Pensamiento Geográfico y Espacio Regional en España. Opu. cit., p. 193

TROITIÑO VINUESA, M. A. (1991): «Centro histórico, intervención urbanística y análisis urbano», Revista Anales de Geografía de la Universidad Complutense, n 11, pp. 25-48.

UZZI, B. (1996): «The sources and consequences of embeddedness for the economic performance of organizations: The network effect», American Sociological Review, $\mathrm{n}^{\mathrm{o}} 61$, pp. 674-698.

UZZI, B. y SPIRO, J. (2005): «Collaboration and Creativity: The Small World Problem», American Journal of Sociology, vol. 111, $\mathrm{n}^{\circ} 2$, pp. 447-504.

VALLS, J.F.(2008): Fenómeno Low Cost. El impacto en el factor precio. Editorial Deusto, Barcelona.

VAN TRIJP, H.CM. y OUDE OPHUIS, P. (1995): «Perceived quality: a market driven and consumer oriented approach», Food Quality and Preference, vol. 6, n 3, pp. 177-183.

WALKER, G., KOGUT, B. y SHAN, W. (1997): «Social capital, structural holes and the formation of an industry network», Organization Science, vol. 8, no 2, pp. 108-125.

WASSERMAN, S. y FAUST K. (1994): Social Network Analysis. Methods and Applications, Cambridge University Press, Cambridge.

WORLD TOURISM ORGANIZATION (2013): UNTWTO World Torurism Barometer 2014.Vol.12, UNWTO, Madrid.

\section{WEBGRAFÍA}

ASOCIACIÓN DE COMERCIANTES DEL BARRIO DE LAS LETRAS (2016), Disponible en: http://www.barrioletras.com/ 
EU RESEARCH ON SOCIAL SCIENCES AND HUMANITIES. Social innovation, governance and community building. SINGOCOM. European Commission (2015), Disponible en: http://ec.europa.eu/research/research-eu

EXCELTUR. UrbanTUR 2012. Monitor de competitividad turística de los destinos urbanos españoles (2012), Disponible en: http://exceltur.org/wp-content/uploads/2014/10/ URBANTUR-2012_INFORME.pdf

IET (2015): Instituto de estudios turísticos; El turismo español en cifras 2014, Disponible en: http://www.iet.tourspain.es/informes/Documentacion/TurEspCifras2014.pdf

\section{ANEXO 1: CUESTIONARIO}

\section{PARTE I}

Pregunta 1. Nombre del comercio

Pregunta 2. Año de apertura

\begin{tabular}{|l|l|l|}
\hline o 1.1980 o anterior & o 13.1992 & o 26.2005 \\
o 2.1981 & o 14.1993 & o 27.2006 \\
o 3.1982 & o 15.1994 & o 28.2007 \\
o 4.1983 & o 16.1995 & o 29.2008 \\
o 5.1984 & o 17.1996 & o 30.2009 \\
o 6.1985 & o 18.1997 & o 31.2010 \\
o 7.1986 & o 19.1998 & o 32.2011 \\
o 8.1987 & o 20.1999 & o 33.2012 \\
o 9.1988 & o 21.2000 & o 34.2013 \\
o 10.1989 & o 22.2001 & o 35.2014 \\
o 11.1990 & o 23.2002 & o 36.2015 \\
o 12.1991 & o 24.2003 & \\
\hline
\end{tabular}

Pregunta 3. Año de incorporación a la asociación:

\begin{tabular}{|l|l|l|}
\hline o 1.1980 o anterior & o 12.1991 & o 25.2004 \\
o 2.1981 & o 13.1992 & o 26.2005 \\
o 3.1982 & o 14.1993 & o 27.2006 \\
o 4.1983 & o 15.1994 & o 28.2007 \\
o 5.1984 & o 16.1995 & o 29.2008 \\
o 6.1985 & o 17.1996 & o 30.2009 \\
o 7.1986 & o 18.1997 & o 31.2010 \\
o 8.1987 & o 19.1998 & o 32.2011 \\
o 9.1988 & o 20.1999 & o 33.2012 \\
o 10.1989 & o 21.2000 & o 34.2013 \\
o 11.1990 & o 22.2001 & o 35.2014 \\
& o 23.2002 & o 36.2015 \\
\hline
\end{tabular}

Pregunta 4. Dirección del local 
Pregunta 5. Sexo del propietario

Pregunta 6. Nacionalidad

Pregunta 7. Cargo

Pregunta 8. o 1. Primarios o 1. Femenino

o 1. Español

o 1. Emprendedor

o 2. Secundarios o 2. Masculino

o 2. No Español

o 2. Empleado

Pregunta 9. Cantidad de empleados

Pregunta 10. Facturación anual

\begin{tabular}{l|l} 
o $1.0-20000 €$ & $\begin{array}{l}\text { o } 4 .+60000 \\
\text { o } 5 . \text { Abrio en 2015, ejercicio no terminado } \\
\text { o } 2.20000-40000 €\end{array}$ \\
o $3.40000-60000 €$ & o. No quiere o no sabe contestar \\
\hline
\end{tabular}

Pregunta 11. Utilización de herramientas de comunicación en Internet

\begin{tabular}{|l|l|}
\hline o 1. Web & o 5. Instagram \\
o 2. Blog & o 6. Twitter \\
o 3. Facebook & o 7. Pinterest \\
o 4. Correo electrónico & o 8. Otros \\
\hline
\end{tabular}

Pregunta 12. Actividad principal comercios

\begin{tabular}{|l|l}
\hline o 1. Antigüedades & o 11. Informática/web \\
o 2. Alimentación & o 12. Joyerias y bisuterias \\
o 3. Arte & o 13. Liberias, papelerias... \\
o 4. Belleza & o 14. Moda. Textil y complementos \\
o 5. Comunicaciones & o 16. Tiempo libre \\
o 6. Flores y plantas & o 17. Otras actividades comerciales \\
o 7. Fotografía & o 18. Reparación y venta motocicletas \\
o 8. Hogar y decoración & o 19. Otro \\
o 9. Impresión. Artes gráficas. & \\
\hline
\end{tabular}

Pregunta 13. Actividad principal hospedaje:

\begin{tabular}{|l|l|}
\hline o 1. Hoteles & $\begin{array}{l}\text { o 3. Pensiones } \\
\text { o } 2 . \text { Hostales }\end{array}$ \\
\hline
\end{tabular}

Pregunta 14. Actividad principal servicios

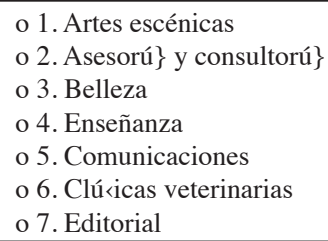

o 8. Entidades financieras

o 9. Gimnasio/Spa

o 10. Inmobiliarias

o 11. Salud. Centros sanitarios

o 12. Servicios Técnicos

o 13. Tiempo libre

o 14. Otros servicios 
Pregunta 15. Actividad principal

\begin{tabular}{|l|l|l|}
\hline o 1. Hostelería & o 2. Instituciones & o 3. Cultura y turismo \\
\hline
\end{tabular}

Pregunta 16. Fidelización turística

\begin{tabular}{|l|l|}
\hline o 1.2009 & o 5.2013 \\
o 2.2010 & o 6.2014 \\
o 3.2011 & o 7.2015 \\
o 4.2012 & \\
\hline
\end{tabular}

Pregunta 17. Nos vamos de BARTES
o 1.2014
o 2.2015

Pregunta 18. Black Friday
o 1.2014
o 2.2015

Pregunta 19. La noche de los libros

\begin{tabular}{|l|l|}
\hline o 1.2010 & o 4.2013 \\
o 2.2011 & o 5.2014 \\
o 3.2012 & o 6.2015 \\
\hline
\end{tabular}

Pregunta 20. Decoración

\begin{tabular}{|l|l|}
\hline o 1.2010 & o 4.2013 \\
o 2.2011 & o 5.2014 \\
o 3.2012 & o 6.2015 \\
\hline
\end{tabular}

Pregunta 21. Mercado de las Ranas

\begin{tabular}{|l|l|}
\hline o 1.2012 & 03.2014 \\
o 2.2013 & 04.2015 \\
\hline
\end{tabular}

Pregunta 22. Gastro Bacchus: De tapas por el Madrid de los vinos. Barrio de las letras.
o 1.2014
o 2.2015

Pregunta 23. Sintió el impacto en las ventas de las acciones comerciales
o 1. Si
o 2 . No

Pregunta 24. Se anuncia en la revista del Barrio de las Letras
o 1 . Si
o 2. No

Pregunta 25. Menciona en su web que es miembro de la asociación
o $1 . \mathrm{Si}$
o 2 . No 
Pregunta 26. Tiene algún distintivo en el local de que es miembro de la asociación
o $1 . \mathrm{Si}$
o 2 . No

Pregunta 27. Cree que la información de la asociación ha aumentado las ventas de su negocio
o $1 . \mathrm{Si}$
o 2. No

\section{PARTE II}

1) Listar con que otros comercios del barrio (dueños o empleados de los mismos) se relaciona con más frecuencia:

\begin{tabular}{|c|c|c|c|c|c|}
\hline $\begin{array}{c}\mathrm{N}^{\circ} \mathrm{de} \\
\text { comercio }\end{array}$ & $\begin{array}{c}\text { Muy fuerte } \\
\text { (8 o mas } \\
\text { encuentros) }\end{array}$ & $\begin{array}{c}\text { Fuerte (7-5 } \\
\text { encuentros/ } \\
\text { mes) }\end{array}$ & $\begin{array}{c}\text { Normal (3-4 } \\
\text { encuentros/ } \\
\text { mes) }\end{array}$ & $\begin{array}{c}\text { Débil } \\
\text { (2 encuentros/ } \\
\text { mes) }\end{array}$ & $\begin{array}{c}\text { Muy débil (un } \\
\text { encuentro/ } \\
\text { mes) }\end{array}$ \\
\hline & & & & & \\
\hline & & & & & \\
\hline & & & & & \\
\hline & & & & & \\
\hline & & & & & \\
\hline & & & & & \\
\hline & & & & & \\
\hline & & & & & \\
\hline
\end{tabular}

2) Listar las relaciones con otros comercios del barrio (dueños o empleados de los mismos) son más relevantes para la sobrevivencia de su comercio:

\begin{tabular}{|c|c|c|c|c|c|}
\hline $\begin{array}{c}\mathrm{N}^{\mathrm{o}} \mathrm{de} \\
\text { comercio }\end{array}$ & $\begin{array}{l}\text { Muy fuerte } \\
\text { (8 o más } \\
\text { encuentros) }\end{array}$ & $\begin{array}{c}\text { Fuerte (7-5 } \\
\text { encuentros/ } \\
\text { mes) }\end{array}$ & $\begin{array}{c}\text { Normal (3-4 } \\
\text { encuentros/ } \\
\text { mes) }\end{array}$ & $\begin{array}{c}\text { Débil } \\
\text { (2 encuentros/ } \\
\text { mes) }\end{array}$ & $\begin{array}{c}\text { Muy débil (un } \\
\text { encuentro/ } \\
\text { mes) }\end{array}$ \\
\hline & & & & & \\
\hline & & & & & \\
\hline & & & & & \\
\hline & & & & & \\
\hline & & & & & \\
\hline & & & & & \\
\hline & & & & & \\
\hline & & & & & \\
\hline
\end{tabular}


3) Liste en primer lugar a todas las personas/comercios que ha mencionado anteriormente, mantenga el mismo orden al listarlas horizontalmente. Posteriormente, indique a quién conoce indicando con una $\mathrm{x}$ en la cuadrícula correspondiente a la intersección de las dos personas/comercios. Deje la celda en blanco si estas personas/comercios no tienen ninguna relación o si usted ignora si realmente existe.

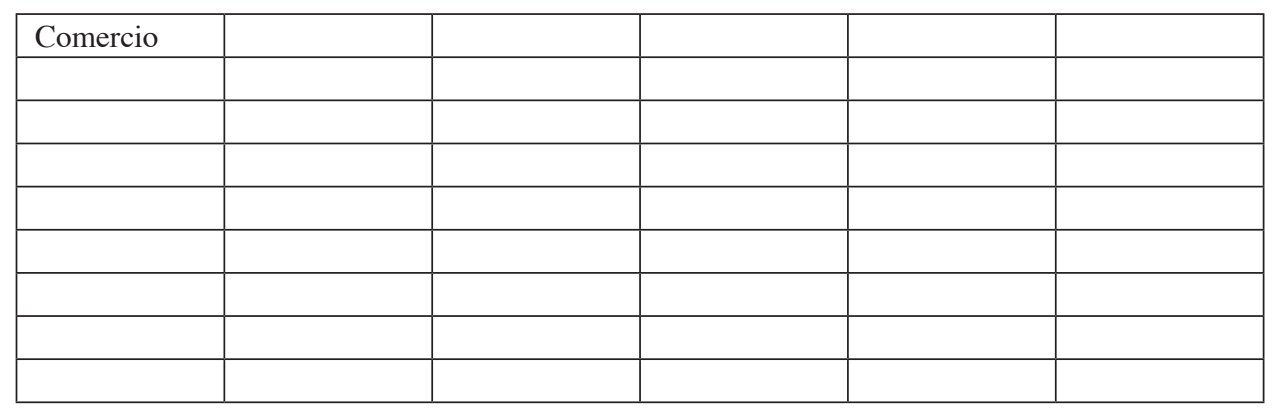

